\title{
Germline acquisition of Cas9/RNA-mediated gene modifications in monkeys
}

\author{
Cell Research (2015) 25:262-265. doi:10.1038/cr.2014.167; published online 23 December 2014
}

\section{Dear Editor,}

Precise gene targeting in monkeys will substantially facilitate generation of human disease models $[1,2]$. Although several transgenic monkey models have been successfully generated [3-6], most of past endeavors in gene targeting using primates had unfortunately failed. The usage of reprogrammable endonucleases, such as ZFN (zinc-finger nuclease), TALEN (transcription activator-like effector nuclease) and CRISPR (clustered regularly interspaced short palindromic repeat)/Cas (CRISPR-associated) system, has opened new avenues for precise gene modification in monkeys [1, 2]. Among these, the CRISPR/Cas9 system has shown to be most efficacious in gene targeting based on its successful application in a number of animal species $[7,8]$.

Recently we reported our success in CRISPR/ Cas9-mediated precise gene targeting in monkeys [9]. The Cas9 nuclease is targeted to a specific genetic locus by a small guide RNA (sgRNA) via base pairing. The CRISPR/Cas9 system is known to be simple, highly specific and efficient, as well as easily adaptable for simultaneous multiple site-specific genomic engineering [10]. Through microinjection of Cas 9 mRNA and sgRNAs targeting 3 genes, i.e., $\mathrm{NrOb1}$ (nuclear receptor subfamily 0 group b member 1$)$, Ppar- $\gamma$ (peroxisome proliferator-activated receptor- $\gamma$ ), and Ragl (recombination activating gene 1), into one-cell embryos, 10 pregnancies were established from 29 surrogate cynomolgus monkeys (Macaca fascicularis). The first set of full-term delivered twin female infants harbored mutations of Ppar- $\gamma$ and Ragl but not $\mathrm{NrObl}$ without any detectable off-target effects [9]. Monkey genome manipulation using TALEN has been also reported [11]. These successes demonstrate the feasibility of precise gene targeting in monkeys.

However, to establish genetically modified monkey colony, germline transmission of genetic modifications is required. While successful germline transmission of transgenes had been reported in monkeys [5], it remains to be determined whether CRISPR/Cas9-mediated genome editing in monkeys can be transmitted to the next generation. As CRISPR/Cas9-mediated genome modifications have been reported to achieve germline transmission in other species, including zebrafish, C. elegans, Drosophila, mouse, rat, etc. [7], germline transmission of Cas9-mediated precise genome modifications in monkeys is highly possible. In our previous study, a pair of twin female monkeys with Ppar- $\gamma$ and Ragl mutations were born [9]. As it takes about 3-4 years for monkeys to reach sexual maturity, we currently cannot perform breeding to assess germline transmission. In this study, we set out to examine gene targeting in gonads and germ cells to explore whether germline transmission could happen in our Cas9-manipulated monkeys.

The total 8 pregnancies with Cas9-manipulated embryos [9] comprised 4 triplets, 1 twin, and 3 singlets (Supplementary information, Table S1). Among them, three live infant founders (Supplementary information, Figure S1A), including two male (founders I and J) and 1 female monkey (founder N), were successfully delivered from 2 singlet and 1 triplet pregnancies at full term either naturally (I) or through caesarean section ( $\mathrm{J}$ and $\mathrm{N})$. One female infant (fetus $\mathrm{H}$ ) from the third singlet pregnancy died after birth. The rest 4 pregnant monkeys all went through miscarriage in different gestation stages, including one twin on day 116 (fetuses F and G), three triplets on day 84 (fetuses were not collected), 95 (fetuses C, D and $\mathrm{E}$ ), and 122 (fetuses $\mathrm{K}, \mathrm{L}$ and $\mathrm{M}$ ), respectively. Except for the triplet miscarried on day 84 , all other 8 aborted embryos, including 6 males (fetuses C-F, L and M) and 2 females (fetuses $\mathrm{G}$ and $\mathrm{K}$ ), and the aforementioned dead female infant (fetus H) were collected (Supplementary information, Table S1). It is worth noting that Cas9 injection led to compatible pregnancy rate but low birth rate [12]. Considering that the cynomolgus macaque is a monotocous species, multiple pregnancies may affect pregnancy success. Indeed, of the 8 pregnancies, all 3 singlets were delivered at full term, whereas all multiple pregnancies, except for one triplet, went through miscarriage (Supplementary information, Table S1). Even though a selective foeticide procedure was applied to 3 triplet pregnancies (recipient No. 070092, 070220 and 
080260) to surgically destroy extra embryos to ensure pregnancy success, only one pregnancy went to full term with the birth of a healthy baby, and the other two failed and were aborted (Supplementary information, Table S1).
We previously reported the analyses of limited collectable tissues from the aforementioned 2 live monkeys [9]. With the aborted embryos, we were able to determine the integration of Cas9-mediated modifications in

A

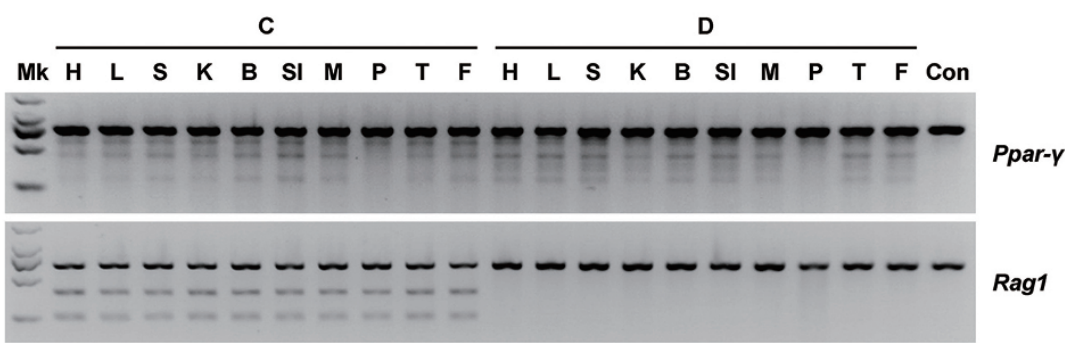

B

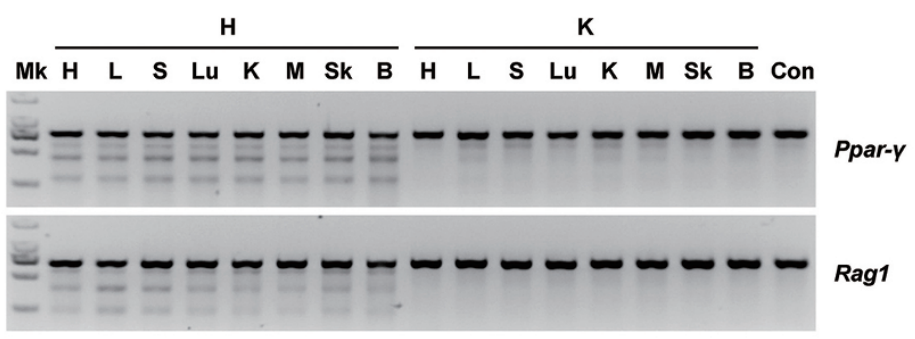

C Ppar- $\gamma$ CCCACTCCTTTGACATCAAGCCCTTCACTACTGTTGACTTCTCCAGCATTTCTGCTCCACATT (WT) C\&D\&H\&K CCCACTCCTTTGACATCAAGCCCTTCACTACTGTTGACTTCTCCAGCATTTCTGCTCCACATT (+1)

Rag1 TTCCGCTATGATTCAGCTTTGGTGTCTGCTTTGATGGACATGGAAGAAGACATCTTGGAAGGC (WT)

C TTCCGCTATGATTCAGCTTTGGTGTCTGCTTTG: : : : : :ATGGAAGAAGACATCTTGGAAGGC (-6)

H TTCCGCTATGATTCAGCTTTGGTGTCTGCTT: : : : : : : : : : : AAGAAGACATCTTGGAAGGC (-12)

D

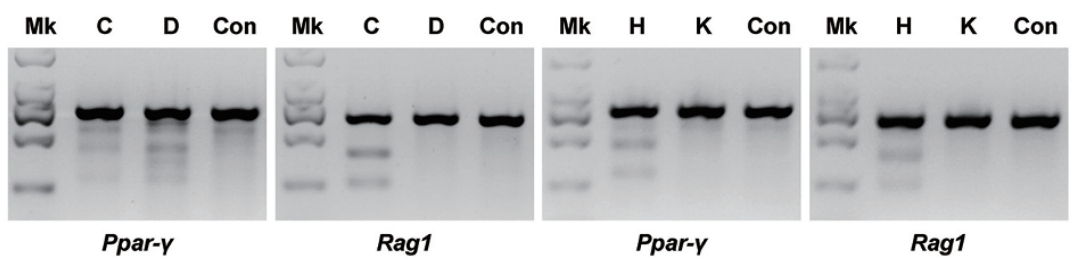

E Ppar- $\gamma$ CCCACTCCTTTGACATCAAGCCCTTCACTACTGTTGACTTCTCCAGCATTTCTGCTCCACATT (WT)

D CCCACTCCTTTGACATCAAGCCCTTCACTACTGTTGACTTCTCCAGCATTTCTGCTCCACATT $(+1,2 / 30)$

$\hat{a}$

H CCСАCTCCTTTGACATCAAGCCCTTCACTACTGTTGACTTCTCCAGCATTTCTGCTCCACATT $(+1,8 / 22)$

Rag1 TTCCGCTATGATTCAGCTTTGGTGTCTGCTTTGATGGACATGGAAGAAGACATCTTGGAAGGC (WT)

C TTCCGCTATGATTCAGCTTTGGTGTCTGCTTTG : : : : : : ATGGAAGAAGACATCTTGGAAGGC $(-6,5 / 15)$

H TTCCGCTATGATTCAGCTTTGGTGTCTGCTT: : : : : : : : : : : : AAGAAGACATCTTGGAAGGC $(-12,3 / 23)$

$\mathbf{F}$

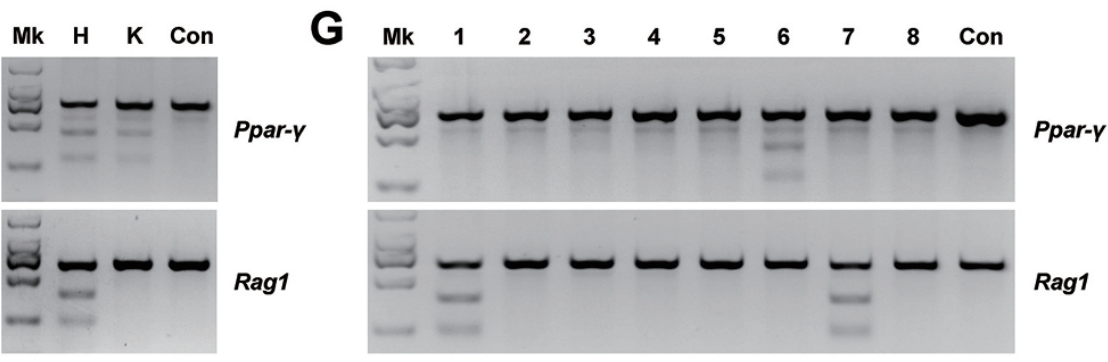

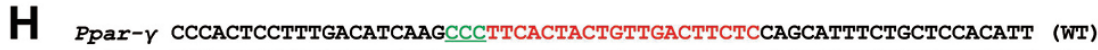

\#6 CCСАСTCCTTTGACATCAAGCCCTTCACTACTGTTGACTTCTCCAGCATTTCTGCTCCACATT $(+1,9 / 19)$

Rag1 TTCCGCTATGATTCAGCTTTGGTGTCTGCTTTGATGGACATGGAAGAAGACATCTTGGAAGGC (WT)

\#1 TTCCGCTATGATTCAGCTTTGGTGTCTGCTT: : : : : : : : : :: :AAGAAGACATCTTGGAAGGC $(-12,6 / 13)$

\#7 TTCCGCTATGATTCAGCTTTGGTGTCTGCTT : : : : : : : : : : : : :AAGAAGACATCTTGGAAGGC $(-12,8 / 16)$ 
Figure 1 sgRNA:Cas9-mediated modifications in somatic tissues and germ cells of aborted fetuses. (A) Detection of the sgRNA:Cas9-mediated on-target cleavage of Ppar- $\gamma$ and Rag1 by T7EN1 cleavage assay in aborted fetuses $\mathrm{C}$ and D. PCR products were amplified and subjected to T7EN1 cleavage assay. H, heart; L, liver; S, spleen; K, kidney; B, brain; SI, small intestine; M, skeletal muscle; P, placenta; T, tail; F, finger; Con, wild-type control; Mk, marker. (B) Detection of the sgRNA:Cas9-mediated on-target cleavage of Ppar- $\gamma$ and Rag1 by T7EN1 cleavage assay in aborted fetuses $\mathrm{H}$ and K. Lu, lung; Sk, skin. (C) DNA sequencing results of fetuses C, D, H, and K. TA clones from PCR products were analyzed by DNA sequencing. The PAM sequences are underlined and highlighted in green; the targeting sequences in red; and mutations in blue, lower case; deletions (-), and insertions (+). (D) Detection of sgRNA:Cas9-mediated on-target cleavage of Ppar- $\gamma$ and Rag1 by T7EN1 cleavage assay in testes of aborted fetuses $C$ and D, and in ovaries of aborted fetuses $\mathrm{H}$ and K. (E) DNA sequencing results of D. No Ppar- $\gamma$ mutant clone was detected in a total of 27 colonies of fetus C. (F) Detection of sgRNA:Cas9-mediated on-target cleavage of Ppar- $\gamma$ and Rag1 by T7EN1 cleavage assay in 2 pools of 7 single germ cells from ovaries of fetuses $\mathrm{H}$ and K. (G) Detection of sgRNA:Cas9-mediated on-target cleavage of Ppar- $\gamma$ and Rag1 by T7EN1 cleavage assay in 8 individual germ cells from the ovary of fetus H. (H) DNA sequencing results of $\mathbf{G}$.

different tissues of monkey embryos, and provide direct evidence to support the conclusion that mutations were wide-spread across different tissues. Given that no $\mathrm{NrObl}$ mutation was detected in all live monkeys, we focused our analyses on targeted modifications of Ppar- $\gamma$ and Rag1. By PCR amplification, T7EN1 cleavage assay, and sequencing, we extensively analyzed targeted mutagenesis in 10 somatic tissues, including heart, liver, spleen, kidney, brain, small intestine, skeletal muscle, placenta, tail and finger, from the two first aborted fetuses $\mathrm{C}$ and D. T7EN1 assay showed cleavage bands of Ppar- $\gamma$ in every tissue of both fetuses $C$ and $D$ except for the placenta (Figure 1A). Cleavage bands of Ragl were also observed in every tissue from fetus $\mathrm{C}$, indicating that Cas9-mediated mutation could occur in various tissues derived from all three germ layers. Targeted mutations in different tissues were further confirmed by sequencing analysis (Figure 1C and data not shown), demonstrating the extensive integration of Cas9-mediated gene editing. Targeted mutagenesis was also observed across various tissues in other aborted embryos (fetuses E-H and K-M; Figure 1B and Supplementary information, Figure S1DS1G).

In sum, targeted mutagenesis was detected in 8 out of 9 aborted fetuses with efficiency of 7/9 for Ppar- $\gamma$ (fetuses C, D, E, H, K, L and M), and 4/9 for Ragl (fetuses C, E, $\mathrm{G}$ and $\mathrm{H}$ ). Further analysis revealed that 3 out of 9 fetuses harbor mutations for both Ppar- $\gamma$ and Ragl (fetuses C, $\mathrm{E}$ and $\mathrm{H}$; Figure 1A, 1B and Supplementary information, Figure S1D and S1E), demonstrating the potency of the CRISPR/Cas9 method in monkeys.

Meanwhile, as described before [9], Cas9-mediated targeted mutagenesis of Ppar- $\gamma$ and Ragl was carefully analyzed in the placenta, umbilical cord and ear punch tissues of the 3 live infant monkeys. The results indicated that no targeted mutagenesis was detected in founders I and J. The cleavage bands of Ragl, but not Ppar- $\gamma$, were observed in the placenta of founder $\mathrm{N}$ in the T7EN1 cleavage assay (Supplementary information, Figure S1B). Targeted mutations were further confirmed by se- quencing of PCR products (Supplementary information, Figure S1C). These results provide further evidence to confirm the feasibility of using the CRISPR/Cas9 system for monkey gene targeting. As observed before [9], multiple genotypes of Ppar- $\gamma$ were detected in founder E (Supplementary information, Figure S1D and S1E), further confirming the mosaicism of Cas9-mediated genome targeting in monkeys.

The efficient integration of targeted mutations into different tissues strongly suggests the possibility of gene editing in the gonads. To test this, the testes were isolated from the 2 aborted male embryos $\mathrm{C}$ and $\mathrm{D}$, and the targeted mutagenesis was analyzed by the T7EN1 assay. Consistent with the observations in other somatic tissues, the cleavage of Ragl occurred in the testes from fetus $\mathrm{C}$, and the cleavage of Ppar- $\gamma$ was observed in the testes from both fetuses $\mathrm{C}$ and $\mathrm{D}$ (Figure 1D), demonstrating that Cas9-mediated genome modification was successfully integrated into gonads of the fetuses.

The findings in the males encouraged us to assess the outcome in the females. The ovaries of the aborted female embryos $\mathrm{H}$ and $\mathrm{K}$ were isolated for analysis of targeted mutagenesis. The T7EN1 assay showed cleavage bands of Ppar- $\gamma$ and Ragl in ovaries of fetus H (Figure 1D), which were also observed in various somatic tissues of fetus H (Figure 1B). These data confirm that Cas9-mediated genome modification was integrated into both male and female gonads with efficiency of $3 / 4$ of the tested fetuses.

Targeted mutations were further confirmed by sequencing, which indicated that targeted mutations in the testis, as well as the ovary were the same as those detected in somatic tissues, such as a 1-bp insertion of Ppar- $\gamma$, a 6-bp deletion of Ragl in the testes, as well as a 1-bp insertion of Ppar- $\gamma$, and a 12-bp deletion of Rag1 in the ovaries. No mutation was detected in a total of 27 colonies in Ppar- $\gamma$ locus of fetus C, which showed weak T7EN1 cleavage bands (Figure 1E and data not shown). Also, sequencing results showed Cas9-mediated gene targeting in the gonads with different efficiencies. In the tes- 
tes, the mutagenesis efficiencies were $6.67 \%$ for Ppar- $\gamma$ in fetus D (2/30), and $33.33 \%$ for Rag1 in fetus C (5/15); in the ovaries, the targeting efficiencies were $36.36 \%$ for Ppar- $\gamma(8 / 22)$ and $13.04 \%$ for Ragl in fetus H (3/23; Figure $1 \mathrm{E})$. The fact that targeted mutations were extensively integrated into gonads strongly suggests a potential that Cas9-mediated targeting could occur in germ cells.

To evaluate this potential, germ cells were isolated from ovaries of aborted embryos $\mathrm{H}$ and $\mathrm{K}$, and confirmed by immunostaining with Mvh, a germ cell specific marker [13] (Supplementary information, Figure S1H and S1I). Subsequently, two populations of 7 single germ cells from each aborted fetus were used for detection of mutations. Consistently, T7EN1 cleavage bands of Rag1 were observed in germ cell population from embryo $\mathrm{H}$, and cleavage bands of Ppar- $\gamma$ were detected in germ cell populations from both embryos $\mathrm{H}$ and $\mathrm{K}$ (Figure $1 \mathrm{~F}$ ). The sequencing results further confirmed that these germ cells harbored the same mutations as somatic cells, such as a 1-bp insertion in Ppar- $\gamma(5 / 24$ in fetus $\mathrm{H}, 2 / 14$ in fetus K), and a 12-bp deletion in Ragl (16/23 in fetus $\mathrm{H}$; data not shown). These data provide preliminary evidence that Cas9-mediated monkey gene modifications occurred in germ cells. Remarkably, both tested germ cell populations from 2 different fetuses harbored targeted mutations, demonstrating a high probability of targeted mutations occurring in germ cells.

More supportive evidence was obtained through analysis of individual germ cells. After amplification of the whole genomes from 8 individual germ cells from embryo $\mathrm{H}$, the amplified products were subjected to mutation analyses as described above. Cleavage bands of Ppar- $\gamma$ or Ragl were founded in one and two of the 8 reactions of the T7EN1 assay, respectively (Figure 1G), suggesting that targeted mutations occurred in individual germ cells. Sequencing data further confirmed targeted mutations, such as a 1-bp insertion of Ppar- $\gamma$, and a 12bp deletion of Ragl, which are the same as those detected in the somatic cells and germ cell populations of fetus $\mathrm{H}$ (Figure $1 \mathrm{H}$ ). The targeting efficiency is about $50 \%$, suggesting that mutations likely occur at only one allele in diploid germ cells (Figure 1H). Taken together, targeted mutagenesis detected in the gonad, germ cell population, and single germ cell strongly suggest the possibility of germline transmission of Cas9-mediated genome modifications in monkeys.

In summary, our follow-up studies further confirm the feasibility of using the CRISPR/Cas9 system for gene editing in monkeys. Furthermore, our data demonstrate that Cas9-mediated mutagenesis extensively occurred in various somatic tissues as well as gonads. Most importantly, our study provides imperative evidence at both population and single cell levels that Cas9-mediated gene modifications occur in monkey germline successfully, suggesting that it is highly likely that these modifications could be transmitted through monkey germlines to the next generation. Nevertheless, direct evidence for germline transmission is yet to be obtained.

\section{Acknowledgments}

We thank Dr Ke Tang (Nanchang University) and Dr Yi E Sun (University of California, Los Angeles) for careful reading and editing of the manuscript. This study was supported by the National Basic Research Program of China (2011CB944301 and 2012CBA01300), and the National High Technology Research and Development Program of China (2012AA020701).

Yongchang Chen ${ }^{1,5,6, *}$, Yiqiang $\mathrm{Cui}^{2,}{ }^{*}$, Bin $\mathrm{Shen}^{2,3, *}$, Yuyu Niu ${ }^{1,5,6, *}$, Xiaoyang Zhao ${ }^{4}$, Lei Wang ${ }^{2}$,

Jianying Wang ${ }^{2}$, Wei $\mathrm{Li}^{4}$, Qi Zhou ${ }^{4}$, Weizhi $\mathrm{Ji}^{1,5,6}$, Jiahao Sha ${ }^{2}$, Xingxu Huang ${ }^{3,7}$

${ }^{I}$ Yunnan Key Laboratory of Primate Biomedical Research, Kunming, Yunnan 650500, China; ${ }^{2}$ State Key Laboratory of Reproductive Medicine, Department of Histology and Embryology, Nanjing Medical University, Nanjing, Jiangsu 210029, China $;^{3}$ MOE Key Laboratory of Model Animal for Disease Study, Model Animal Research Center of Nanjing University, National Resource Center for Mutant Mice, Nanjing, Jiangsu 210061, China: ${ }^{4}$ State Key Laboratory of Reproductive Biology, Institute of Zoology, Chinese Academy of Sciences, Beijing 100101, China, ${ }^{5}$ Kunming Biomed International and National Engineering Research Center of Biomedicine and Animal Science, Kunming, Yunnan 650500, China; ${ }^{6}$ Life Science and Technology, Kunming University of Science and Technology, Kunming, Yunnan 650224, China; ' 7 hanghai Key Laboratory of Reproductive Medicine, Shanghai 200025, China

*These four authors contributed equally to this work.

Correspondence: Xingxu Huang ${ }^{\mathrm{a}}$, Jiahao Sha ${ }^{\mathrm{b}}$, Weizhi Ji ${ }^{\mathrm{c}}$

${ }^{a}$ E-mail: xingxuhuang@mail.nju.edu.cn

${ }^{b}$ E-mail: Shajh@njmu.edu.cn

cE-mail: wji@kbimed.com

\section{References}

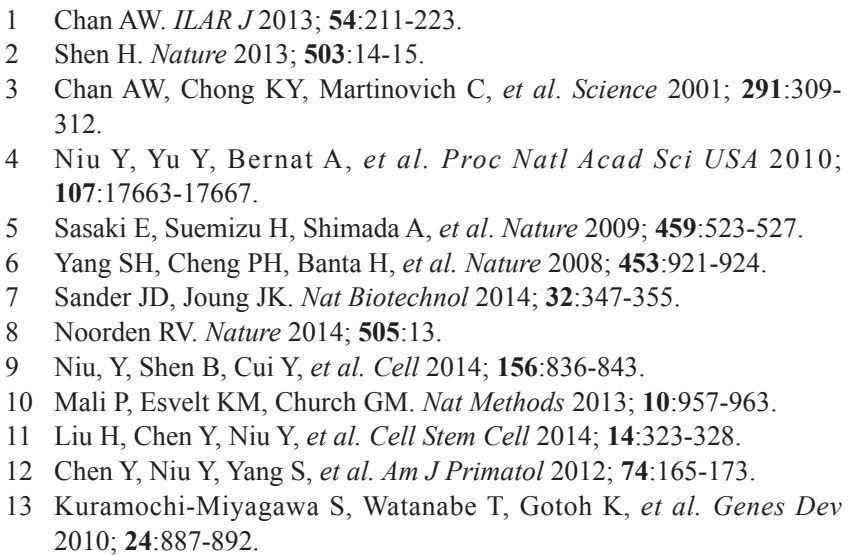

(Supplementary information is linked to the online version of the paper on the Cell Research website.) 